\title{
The Production of CNT Flower-Like Structures with 3-Fold and 4-Fold Symmetries on a Pt/Si Substrate
}

\author{
Madjid Arab $^{1^{*}}$, Marjorie David ${ }^{1}$, Jean-Christophe Valmalette ${ }^{1}$, Christophe Ramseyer ${ }^{2}$ \\ ${ }^{1}$ Université du Sud Toulon Var, IM2NP UMR CNRS, La Garde, France \\ ${ }^{2}$ Université Franche-Comté, UE, La Bouloie, Besançon, France \\ Email: ${ }^{*}$ marjorie.david@univ-tln.fr,madjid.arab@univ-tln.fr
}

Received September 25, 2012; revised October 27, 2012; accepted November 7, 2012

\begin{abstract}
Carbon nanotubes flower (CNTs-F) films were prepared by catalytic chemical vapor deposition (CVD) on a platinum $(\mathrm{Pt})$ thin layer, supported on a silicon wafer. The products were synthesized from an aerosol composed of ferrocene and toluene, as catalyst and carbon precursor respectively, at $820^{\circ} \mathrm{C}$. The high synthesis temperature induces a modification of a Pt thin layer to a nano-structured island giving rise to the formation of CNTs-F during the following films growth step by CVD process. The suggested mechanism involves the selective diffusion of the catalyst and carbon atoms through the Pt grain boundaries. This results in the appearance of flower-like structures with 3-and 4-fold symmetries.
\end{abstract}

Keywords: Carbon Nanotube; Aerosol-CVD; 3-Fold and 4-Fold Symmetries; Flower Shape

\section{Introduction}

Nanomaterials morphology has been paid a great attention for new design and conception at nanoscales. Nevertheless, the carbon nanotubes (CNTs) represent the most performed nanomaterials up to now. Their use requires shape and size control for specific applications. So the preparation of various morphology like: bundles, thin film of CNTs, carpet and forest forms have attracted much interest due to their unique properties and potential applications for electronic, mechanical, gas interaction for storage, sieve and sensing [1-3].

Organization of these nanomaterials on a substrate is crucial, since it is important to control the growth of the aligned carbon nanotubes films on a flat surface. Different surface defects are determined from an extended deposition into the layers. In this case, several growth methods carried out to modify CNTs films. Catalytic chemical vapor deposition (CCVD) has been demonstrated to be a promising technique because of its high purity, simple configuration and flexibility in terms of influential parameters like temperature and the hydrocarbon/precursor ratio. This CCVD methodology avoids the use of a catalyst support and the subsequent support removal procedures. Furthermore, several structures of nanotubes have been reported including 2D and 3D cross-networks based on nanotubes forest, an interesting configuration to build blocks in nanosystems [4-7].

The growth mechanism of these structures depends on

*Corresponding author. the initial growth stage; however, the relationship to the final morphology is still under discussion. In addition, some of the interesting properties such as a low friction and low adhesion involve interfaces compatibilities. This motivates fundamental studies of structure, composition and chemical reactivity.

Here, we report the structural characterization studies of correlations between the formation of CNTs-F on 50 $\mathrm{nm}$ platinum(111) film, supported on a silicon substrates (NEYCO products), from defects and the induced threefold and four-fold symmetry. This structure can be used as a template for growing the pseudo 1D structures along the grooves, with 3- and/or 4-fold symmetries. Interfaces deserve being worthy explored for their ability to produce $2 \mathrm{D}$ or $3 \mathrm{D}$ building components for micro- and nano-electro-mechanical system applications.

\section{Experimental Setup}

\subsection{Carbon Nanotubes Synthesis}

The CNTs films were obtained using aerosol-CCVD combined method $[8,9]$. This approach is based on the catalytic decomposition of a liquid solution by pyrolysing mixed aerosols containing both the hydrocarbon source (toluene) and the catalytic source (ferrocene) which simultaneously and continuously fill the reactor cell. The solution was prepared by dissolving $5 \mathrm{wt} \%$ of ferrocene in toluene.

The substrates maintained in reactor were first directly purged with argon at room temperature at the beginning, 
then the reactor was preheated to preset temperature at $820^{\circ} \mathrm{C}$. To generate the aerosol, we have used a multipiezoelectric system of the ultrasonic generator working at a frequency of $800 \mathrm{kHz}$ and with a power of $100 \mathrm{~W}$. The obtained aerosol was carried towards the high temperature zone of the reactor, by argon with flow rate of 1 $\mathrm{L} \cdot \mathrm{min}^{-1}$, and the decomposition of this vapor took place inside the reactor. At the end of the synthesis, the generator and the furnace were stopped and cooled down to room temperature.

\subsection{Structural Characterization}

The morphologies of CNTs were characterized by scanning electron microscopy (SEM) and transmission electron microscopy (TEM, Philips CM 200, operating at 200) and micro-Raman spectroscopy with $514.5 \mathrm{~nm}$ wavelength excitation. Each sample was first characterized by XRD to identify the phases and to determine the mean size of particles. X-ray diffraction patterns were recorded on a Siemens-Brucker D5000 diffractometer working in a $\theta-2 \theta$ mode, with a copper $\mathrm{X}$-ray source $(\lambda(\mathrm{K} \alpha 1)=$ $\left.1.5406^{\circ} \mathrm{A}\right)$, Soller slides, and secondary monochromator.

\section{Result and Discussion}

The synthesis products allow to obtain the aligned and perpendicularly mutli-walled carbon nanotubes (MWCNTs) on integrated support [8,9]. Powder sample collected on the inner walls of the synthesis reactor are presented in Figure 1(c). The TEM observations of the dispersed nanotubes show different inner and outer mean diameters respectively around $15 \mathrm{~nm}$ and $50 \mathrm{~nm}$. Several nanotubes are opened at the ends and they contained iron catalyst particles (black particles).

SEM imaging (Figure 1(b)) shows a cross section of carbon nanotubes films obtained (etched section) afterwards pyrolysing mixed liquid (toluene/ferrocene) aerosol during $15 \mathrm{~min}$. The aerosol-CCVD method is well known for obtaining the aligned and perpendicularly MWCNTs on an oxide surfaces. MWCNTs film showed a large scale in a few minutes of synthesis. In interesting surfaces for a large scale application (few $\mathrm{mm}^{2}$ surfaces) [10-12]. The aligned growth of MWCNTs on amorphous silica is rather favored by Van Der Waals interactions. The dense structures of aligned CNTs films induce a hexagonal array with 6-fold symmetry.

The CNTs growth synthesis performed on a Pt/Si substrate, gives rise to characteristic "flower-like" with hundred microns size features emerging from a relatively smooth surface (Figure 2(a)). These morphologies can be described as hatching bundles of CNTs with two symmetries types: 4-fold ( $\mathrm{T}$ and square shapes) and 3fold. The branches of the flowers are characterized by large grooves. Both symmetries are present on the same

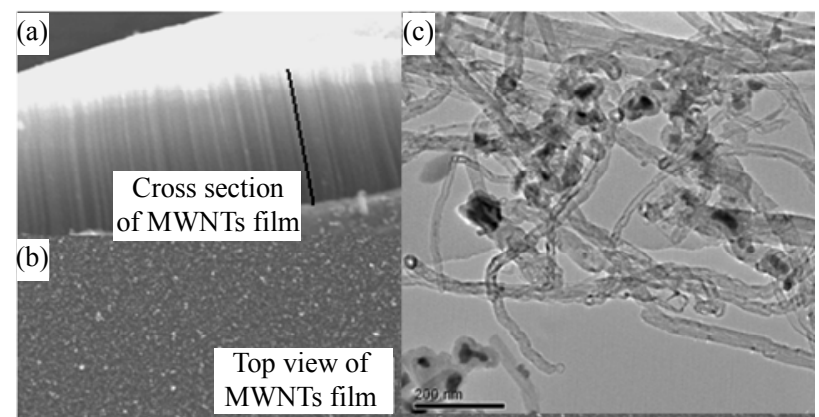

Figure 1. SEM images: (a) Carpet of CNTs on Si; (b) Top view of the MWCNTs film; and (c) TEM image of dispersed MWCNTs.

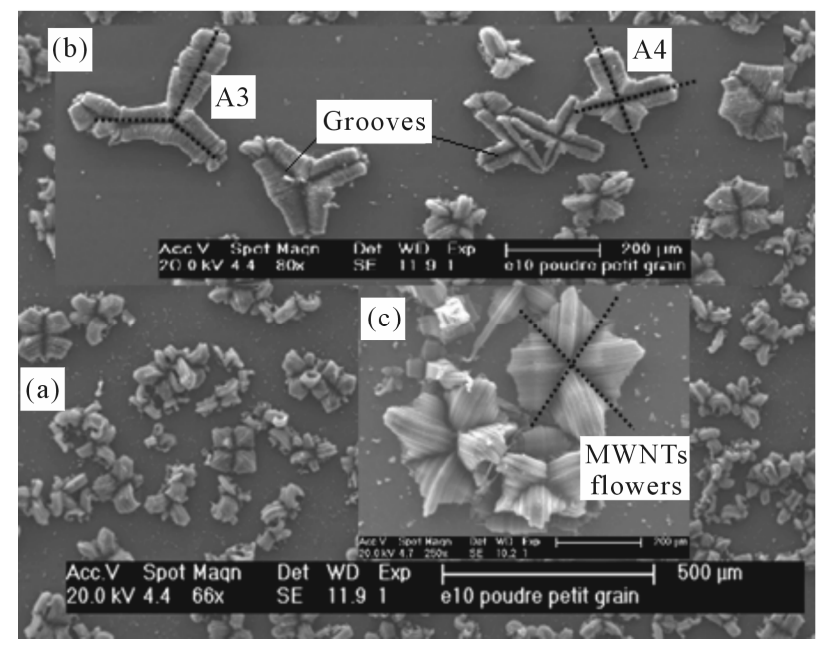

Figure 2. SEM images on top of the MWCNTs film obtained on $\mathrm{Pt} / \mathrm{Si}$ with a package of flowers showing 3-fold and 4-fold symmetries.

sample with mean ratio 1:3 for 3-fold and 4-fold respectively.

Micro Raman spectroscopy analyses are conducted for CNTs collected on the inner walls reactor in powder form and for CNTs-F blooming on the Pt/Si interface (Figure 3(a)). In both samples, we remark the same spectra Raman with identical peaks locations. Each spectrum shows the characteristic $\mathrm{G}$ and $\mathrm{D}$ bands respectively at $1575 \mathrm{~cm}^{-1}$ and $1346 \mathrm{~cm}^{-1}$ and the second order 2D band at $2695 \mathrm{~cm}^{-1}$. These results indicate that MWCNTs are well synthesized. As done in the Figure 3(b), the characterization by X-ray diffraction performed on these films confirm the formation of carbon nanotubes $(2 \theta \approx$ $26.2^{\circ}$ characterizing the presence of graphite) with additional Bragg peaks assigned to platinum layer and iron oxide issued from the used ferrocene catalyst.

To elucidate the flowers growth mechanism, we conducted other experiments on the substrates $(\mathrm{Pt} / \mathrm{Si})$ in the same conditions and without injecting the aerosol mixture liquid in order to analyse the change of the surface state. Figure 4(a) shows the reorganization of the Pt sur- 


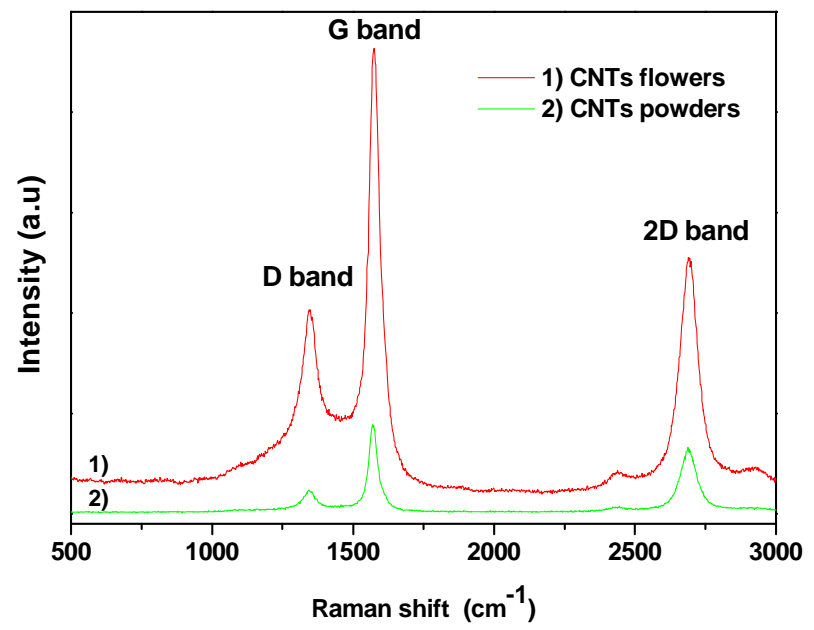

(a)

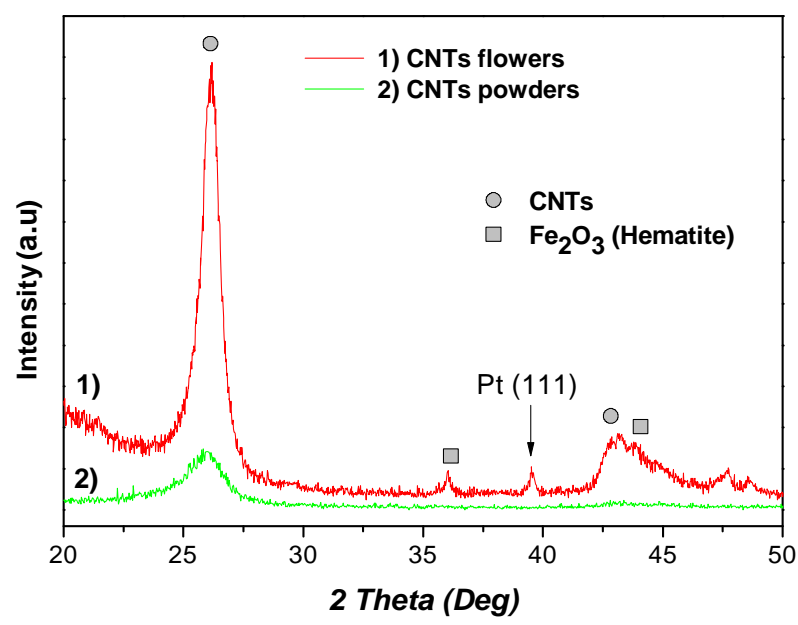

(b)

Figure 3. Powder and flowers forms of CNTs characterization: (a) Raman spectra; and (b) X-ray diffraction pattern.

face on a small islands obtained after 15 minutes at $820^{\circ} \mathrm{C}$. This phenomenon is known following the self organized nanostructures growth which favours the coalescence giving rise to the micro-islands [13]. The morphology of this surface show many defects like as $0 \mathrm{D}$ (point defect) and 1D (grain boundaries junctions).

After introduction of the aerosol in the reactor, the CNTs growth start preferentially on the defects located at the grain boundaries. These areas seem to be thinner after reconstruction which facilitates the atom diffusion. This reconstruction induced defects probably causes the favoured diffusion path throughout the surface, which increase the access of aerosol elements (particularly carbon atoms) toward the $\mathrm{Pt} / \mathrm{Si}$ interface. In this case, the defect as growth template requires particular forms, and the obtained symmetries are favored by substrate defects and CNTs interactions. During the growth process, the flowers blooms, pores and grooves begin to appear (Figure 4(b)) due to the stiffness of carbon nanotubes.

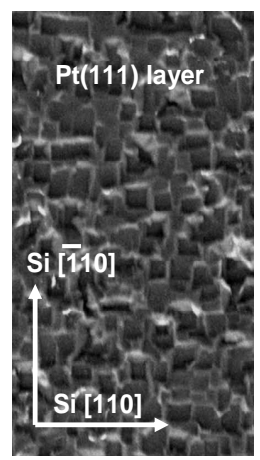

(a)
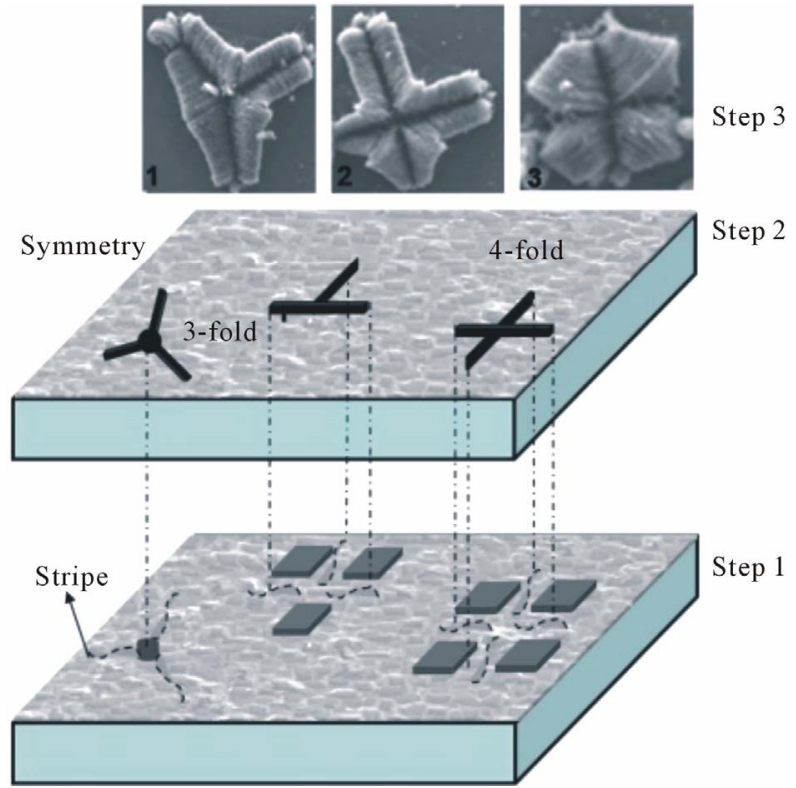

(b)

Figure 4. (a) Annealed $\mathrm{Pt}(111)$ layer on $\mathrm{Si}(001)$ substrate (image size $100 \mu \mathrm{m} \times 200 \mu \mathrm{m}$ ); and (b) Scheme of CNTs-F growth mechanism after $15 \mathrm{~min}$.

\section{Conclusions}

In summary, new forms of CNT flower like structures are obtained on a Pt/Si interfaces by CCVD method at $820^{\circ} \mathrm{C}$. In this study, we note that the reorganization of $\mathrm{Pt}$ layer plays a key role in the growth of CNT-F. The mechanism could involve the defects morphology induced during annealing of $\mathrm{Pt} / \mathrm{Si}$ interfaces. We demonstrated that new 3- and 4-symmetries can be obtained by pre-deposition of a $50 \mathrm{~nm}$ Pt layer heavily reconstructed at CNTs temperature synthesis.

Several studies are in progress to control the defects types during the reorganization of the Pt layer at different temperatures in order to correlate flower symmetries and Pt layer reconstruction at high temperature.

\section{Acknowledgements}

We gratefully acknowledge the Conseil Regional of 
PACA, the Conseil Général du Var, and Toulon Provence Mediterranean for their financial support.

\section{REFERENCES}

[1] R. Saito, G. Dresselhaus and M. S. Dresselhaus, "Physics Properties of Carbon Nanotubes," World Scientific, New York, 1998.

[2] J. Lu and J. Han, "Carbon Nanotubes and NanotubesBased Nano Devices," International Journal of High Speed Electronics and Systems, Vol. 9, No. 1, 1998, pp. 101-123. doi:10.1142/S0129156498000063

[3] M. David, M. Arab, C. Martino, L. Delmas, F. Guinneton and J. R. Gavarri, "Carbon Nanotubes/Ceria Composite Layers Deposited on Surface Acoustic Wave Devices for Gas Detection at Room Temperature," Thin Solid Films, Vol. 520, No. 14, 2012, pp. 4786-4791. doi:10.1016/j.tsf.2011.10.166

[4] S. G. Rao, L. Huang, W. Setyawan and S. H. Hong, "Large-Scale Assembly of Carbon Nanotubes," Nature, Vol. 425, 2003, pp. 36-37. doi:10.1038/425036a

[5] X. L. Li, L. Zhang, X. R. Wang, I. Shimoyama, X. M. Sun, W. S. Seo and H. J. Dai, "Langmuir-Blodgett Assembly of Densely Aligned Single-Walled Carbon Nanotubes from Bulk Materials," Journal of the American Chemical Society, Vol. 129, No. 16, 2007, pp. 4890-4891. doi:10.1021/ja071114e

[6] M. C. LeMieux, M. Roberts, S. Barman, Y. W. Jin, J. M. Kim and Z. N. Bao, "Self-Sorted, Aligned Nanotube Networks for Thin-Film Transistors," Science, Vol. 321, No. 5885, 2008, pp. 101-104. doi:10.1126/science.1156588

[7] W. Zhou, L. Ding, S. Yang and J. Liu, "Orthogonal Ori- entation Control of Carbon Nanotube Growth," Journal of the American Chemical Society, Vol. 132, No. 1, 2010, pp. 336-341. doi:10.1021/ja908414v

[8] M. Arab, F. Berger, F. Picaud, C. Ramseyer, J. Glory and M. Mayne-L'Hermite, "Direct Growth of the Multi-Walled Carbon Nanotubes as a Tool to Detect Ammonia at Room Temperature," Chemical Physics Letters, Vol. 433, No. 13, 2006, pp. 175-181. doi:10.1016/j.cplett.2006.10.036

[9] M. Pinault, M. Mayne-L'Hermite, C. Reynaud, V. Pichot, P. Launois and D. Ballutaud, "Growth of Multiwalled Carbon Nanotubes during the Initial Stages of AerosolAssisted CCVD," Carbon, Vol. 43, No. 14, 2005, pp. 2968-2976. doi:10.1016/j.carbon.2005.06.011

[10] A. Y. Cao, P. L. Dickrel, W. G. Sawyer, M. N. Ghasmeni-Nejhad and P. M. Ajayan, "Super-Compressible Foamlike Carbon Nanotube Films," Science, Vol. 310, No. 5752, 2005, pp. 1307-1308. doi:10.1126/science.1118957

[11] Y. Liu, W. Z. Qian, Q. Zhang, A. Y. Cao, Z. F. Li, W. P. Zhou, Y. Ma and F. Wei, "Hierarchical Agglomerates of Carbon Nanotubes as High-Pressure Cushions," Nano Letters, Vol. 8, No. 5, 2008, pp. 1323-1327. doi: $10.1021 / \mathrm{n} 10733785$

[12] S. B. Hutchens, L. J. Hall and J. R. Greer, "In Situ Mechanical Testing Reveals Periodic Buckle and Propagating in Carbon Nanotubes Bundles," Advanced Functional Materials, Vol. 20, No. 14, 2010, pp. 1-9. doi:10.1002/adfm.201000305

[13] S. Rousset, B. Croset, Y. Girard, G. Prévot, V. Repain and S. Rohart, "Slef-Organized Epitaxial Growth on Spontaneously Nano-Patterned Templates," Comptes Rendus Physique, Vol. 6, No. 1, 2005, pp. 33-46. doi:10.1016/j.crhy.2004.11.010 